\title{
USEFULNESS OF SERUM ELECTROLYTE DETERMINATION IN PATIENTS WITH DEHYDRATION
}

\author{
D. Fahimi, R. Tavakolizadeh, M. Sadeghi \\ Tehran University of Medical Sciences, Tehran, Iran
}

Objectives: Acute gastroenteritis is one of the most common infantile illnesses. Some children have to undergo intravenous fluid therapy based on the severity of dehydration or non-compliance for oral rehydration. The present study aims to assess the effectiveness of routine blood chemical analysis (blood glucose, urea, creatinine, sodium, potassium and bicarbonate) before starting intravenous therapy.

Patients and methods: In the present cross sectional study, all children aged 3 months to 10 years with acute gastroenteritis and without any underlying diseases who underwent intravenous serum therapy in Emergency Department of Bahrami Children Hospital from March 2008 to June 2009 were enrolled into the study. Demographic data, medical history and physical examination of the patients, results of laboratory tests and dehydration grades were noted. Dehydration was categorized according to the criteria approved by World Health Organization. Effectiveness was defined as any change in treatment plan due to laboratory results.

Results: Four hundred and fifty nine children (55.3\% boys, $44.7 \%$ girls, mean age 25.36 months) were assessed. There were statistically significant associations between effectiveness of tests and patients' age, times of diarrhea in the last 24 hours, diarrhea grade and dehydration grade $(p=0.00)$. Only dehydration grade could significantly predict effectiveness according to logistic regression model $(p<0.05)$.

Conclusion: It is not effective to perform blood chemical analysis in all children with acute gastroenteritis. Dehydration grading can be used as a selection criterion for these children. 